\title{
The present day of asteroseismology of $\beta$ Cephei stars: observations
}

\author{
A. Pigulski \\ Instytut Astronomiczny, Uniwersytet Wrocławski, Wrocław, Poland
}

\begin{abstract}
Several successful campaigns on bright $\beta$ Cephei-type pulsators were completed during the recent years yielding detections of many modes at a sub-millimag level. On the other hand, searches for $\beta$ Cephei-type stars in massive photometric databases resulted in a multiplication of the number of known members of this group. These discoveries now allow much better statistical investigations of $\beta$ Cephei stars as a group and a good selection of interesting targets for case studies for asteroseismology.

We summarize the up-to-date achievements of asteroseismology of $\beta$ Cephei stars and present the results of the searches for these stars in the OGLE, ASAS and MACHO databases.
\end{abstract}

\section{Introduction}

The great successes of global helioseismology in modelling the internal structure of the Sun, in particular its internal rotation (see, e.g., Christensen-Dalsgaard 2002 and references therein) led to a growth of interest in applying seismic methods to pulsating stars. The large problems we encounter in this work, however, are due mainly to the low number of detected modes in comparison with millions of modes observed at the solar surface and the difficulty of their proper identification in terms of the quantum numbers used to describe the geometry of pulsations. Since stellar disks are not yet resolved, the cancellation effects limit the detected modes to spherical degrees of $\ell \leq 4$ in photometric data.

From the point of view of asteroseismology, not all types of pulsating stars are equally attractive. Depending on the number of global parameters that define the internal structure of a star, we need to observe a sufficiently large number of modes to constrain these parameters. This explains the advance of asteroseismology in the application to white dwarfs which have rich pulsation spectra consisting of modes that can be relatively easily identified. The observational and theoretical aspects of asteroseismology of different types of pulsating stars across the H-R diagram have been recently reviewed by Kurtz (2006), Handler (2006) and Michel (2006).

A general conclusion from the above-mentioned reviews is that the application of asteroseismology to $\beta$ Cephei stars, massive main-sequence pulsators, looks promising. These stars have relatively simple pulsation spectra, albeit not too simple. Typically, several modes, both radial and non-radial, are observed. Next, many $\beta$ Cephei stars have modes identified and the methods of mode identification developed in the recent years are usually applicable to these stars. Consequently, the sample of $\beta$ Cephei stars studied in detail by means of asteroseismology grows rapidly. We summarize the results of these studies in the next section. It was possible owing to the joint effort of many groups of observers that cooperate in observing the most interesting targets within multisite campaigns.

Presently, the observational studies of $\beta$ Cephei stars follow three main approaches:

- Case study of selected targets consisting usually of a photometric and/or spectroscopic observing campaign followed by detailed asteroseismic modelling.

- Searches for new $\beta$ Cephei stars using mainly massive photometric surveys like OGLE, MACHO or ASAS. 
Table 1: A list of $\beta$ Cephei stars that were the best targets of recent asteroseismic studies. $N_{\text {high }}, N_{\text {low }}$, and $\mathrm{N}_{\text {id }}$ stand for the number of detected high-frequency, low-frequency modes and the number of modes with unambiguous identifications, respectively. As remarks, we indicate when photometric $(\mathrm{PH})$ and spectroscopic (SP) data were used to find and identify modes.

\begin{tabular}{ccccccc}
\hline & & \multicolumn{3}{c}{ References to } & \\
\cline { 3 - 5 } Star & $N_{\text {high }}+N_{\text {low }}$ & $N_{\text {id }}$ & observations & mode id & asteroseis. & Remarks \\
\hline V836 Cen & $6+0$ & 6 & $(1)$ & $(2),(3)$ & $(2),(3)$ & $\mathrm{PH}$ \\
$\nu$ Eri & $12+2$ & 11 & $(4),(5),(7)$ & $(6)-(8)$ & $(8),(9)$ & $\mathrm{PH}+\mathrm{SP}$ \\
12 Lac & $10+2$ & 5 & $(10)$ & $(10)$ & - & $\mathrm{PH}$ \\
$\theta$ Oph & $7+0$ & 3 & $(11),(12)$ & $(11),(12)$ & - & $\mathrm{PH}+\mathrm{SP}$ \\
\hline
\end{tabular}

References: (1) - Aerts et al. (2004b), (2) - Aerts et al. (2003), (3) - Dupret et al. (2004), (4) - Handler et al. (2004), (5) - Aerts et al. (2004a), (6) - De Ridder et al. (2004), (7) - Jerzykiewicz et al. (2005), (8) - Pamyatnykh et al. (2004), (9) - Ausseloos et al. (2005), (10) - Handler et al. (2006), (11) - Handler et al. (2005), (12) - Briquet et al. (2005).

- Observations of $\beta$ Cephei stars in environments that have different chemical abundances, in particular, in Large and Small Magellanic Clouds and different regions of the Galaxy, including the Galactic field and young open clusters.

In the subsequent sections, we will summarize the recent achievements of these three approaches.

\section{Case study of selected targets}

The main requirement for successful seismic modelling is the proper mode identification. Since there are some factors that can help to achieve this, we list them here as they are important in the context of selecting targets for the follow-up study. These helpful factors are the following: (i) the presence of a large number of modes, (ii) the presence of rotational splitting(s), (iii) large amplitudes, (iv) cluster membership. In addition, if a pulsating star is a component of an eclipsing and/or spectroscopic binary, some global parameters can usually be better constrained.

For this reason, stars that show a large number of modes, especially such modes which are close in frequency and therefore suspected to be rotationally split, are preferred during the selection of targets for a detailed seismic study. Table 1 lists basic information for four stars that either were the subject of recent asteroseismic studies (V836 Cen, $\nu$ Eri) or the asteroseismic study is in progress for them (12 Lac, $\theta \mathrm{Oph})$. The schematic frequency spectra of these four stars are shown in Fig. 1.

More $\beta$ Cephei stars ( $\delta$ Cet, $\beta$ CMa, SY Equ) were observed recently, but they have a smaller number of modes detected or identifications are not available for some modes which means that the information that can be obtained from modelling these stars is rather limited.

A lesson learnt from the first decade of asteroseismology of $\beta$ Cephei stars is that we still need to study more stars before some general conclusions concerning the interiors of early B-type stars can be drawn. There is some indication of non-rigid rotation (faster in the core) coming from the studies of Dupret et al. (2004) and Pamyatnykh et al. (2004), but there are still problems in matching frequencies of all modes with a consistent model on the one hand and making them unstable on the other. If we really want to get such information as metallicity, overshooting parameter, mass, age, and rotation in the core from seismic modelling, we really need to have more modes in a single star detected and identified. 


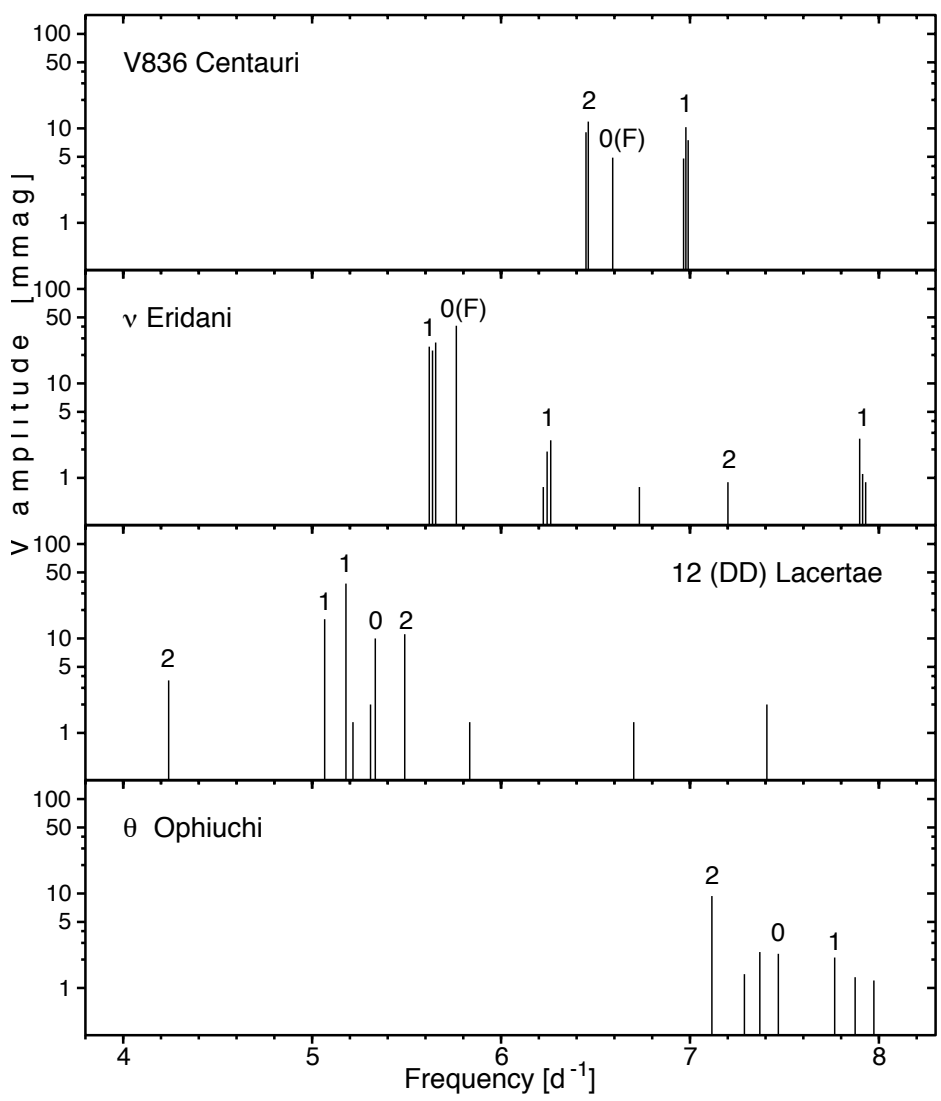

Figure 1: Schematic frequency spectra of four $\beta$ Cephei stars with the largest number of detected modes. Modes that have their spherical degree $\ell$ identified are labelled with the corresponding $\ell$ value.

Fortunately, new observing campaigns were initiated. In particular, three open clusters, NGC 3293 (Handler et al. 2007), NGC 6910 and $\chi$ Persei (Pigulski et al. 2007) were selected as targets in the ongoing campaigns. We already know over $20 \beta$ Cephei stars in these clusters. A preliminary analysis of the star WEBDA 18 , a member of NGC 6910, already resulted in the detection of seven modes, but we can expect that more will be found in the final analysis. Additional constraints on the global parameters of the cluster members will surely help in their seismic modelling.

\section{Searches in massive photometric surveys}

Soon after the publication of the review paper on $\beta$ Cephei stars by Stankov \& Handler (2005), listing 93 certain members of this group, 19 new $\beta$ Cephei stars were found by Pigulski (2005) and Handler (2005) in the published photometry of the All Sky Automated Survey (ASAS, Pojmański 2001). The main goal of this survey is to monitor the whole sky 
for variability. About $70 \%$ of the sky south of declination $+28^{\circ}$ is already monitored. The observations cover stars in the magnitude range between 7 and $14 \mathrm{mag}$ in $\mathrm{V}$. In a series of papers (Pojmański et al. 2005 and references therein), a catalogue of about 50000 variable stars was published. All but two of these 19 new stars have semi-amplitudes larger than $35 \mathrm{mmag}$ which is a rather large value for $\beta$ Cephei stars. This was the consequence of using the dispersion vs. magnitude diagram for the selection of stars in the catalogue. On the other hand, the detection threshold in Fourier spectra for the brightest observed stars amounted to about 3-5 mmag. It became obvious that a large number of $\beta$ Cephei stars could be discovered once the whole database of over $10^{7}$ stars observed within the ASAS survey will be analysed by means of Fourier analysis.

In the first step, we decided to select $\mathrm{O}$ and $\mathrm{B}$ stars from the existing catalogues and then analyse their ASAS photometry. The best way to select OB stars (and distinguish them from $\delta$ Scuti stars which partly overlap with $\beta$ Cephei stars in period) is to use their spectral types and/or UBV photometry. A very homogeneous catalogue of spectral types for bright southern objects is the Michigan Spectral Survey catalogue published by N. Houk and her collaborators in five volumes (Houk \& Swift 1999 and references therein). The catalogue provides MK spectral types for stars with HD numbers south of declination $+5^{\circ}$. About $4,000 \mathrm{OB}$ stars were selected from this catalogue. The sample is homogeneous and magnitude-limited. As a result of the analysis of the ASAS V-filter photometry of this sample, we found 102 new $\beta$ Cephei stars (Pigulski \& Pojmański, in preparation).

A second sample of about 11,000 OB stars was selected from over 200 other catalogues. The analysis of this sample is underway; a preliminary check of the results indicates that we can expect to find another 100-150 new $\beta$ Cephei stars.

This finding triples the number of known $\beta$ Cephei stars and shows the potential of all-sky surveys like ASAS in studying all types of variable stars. Figure 2 shows the amplitudes of the main modes for the $220 \beta$ Cephei stars that are currently known of which more than half were found using ASAS photometry. As mentioned above, this number will soon increase to over 300 , once the analysis of the second sample of the ASAS data will be completed.

It is also obvious that in the sample of 102 newly-discovered $\beta$ Cephei stars we will find excellent targets for follow-up asteroseismic studies, as all these stars are bright; with a few exceptions, they fall in the range between 8 and 10 mag in V. Going back to the discussion on the selection of objects suitable for asteroseismology, we have in this sample:

- Seven stars with modes equidistant (or almost equidistant) in frequency, presumably due to rotational splitting. In two stars, quadruplets, and in the other five stars, triplets were detected. As far as we are aware, these are the first $\beta$ Cephei stars in which quadruplets are seen. This leads to the suspicion that we observe rotationally split $\ell$ $=2$ mode in which one component was not detected. Since the detection threshold for these stars in the ASAS data is still high (3-5 mmag), follow-up campaigns should easily reveal more modes.

- Many stars with large amplitudes. As can be seen from Fig. 2, over 20 stars discovered with ASAS have semi-amplitudes of the main mode larger than $30 \mathrm{mmag}$. These are mostly the stars discovered by Pigulski (2005) and Handler (2005), but in the discussed sample, another large-amplitude star, HD 173006, was found.

- Many stars belonging to open clusters and OB associations. Although the ASAS photometry is not suitable for finding variable stars in clusters owing to its poor spatial resolution, we find some variables in some loose open clusters and in the outer regions of denser ones.

- Four stars that are presumably the primary components of eclipsing binaries. Two, maybe three, are suspected to be double-lined spectroscopic binaries which will allow to derive their stellar parameters directly. 


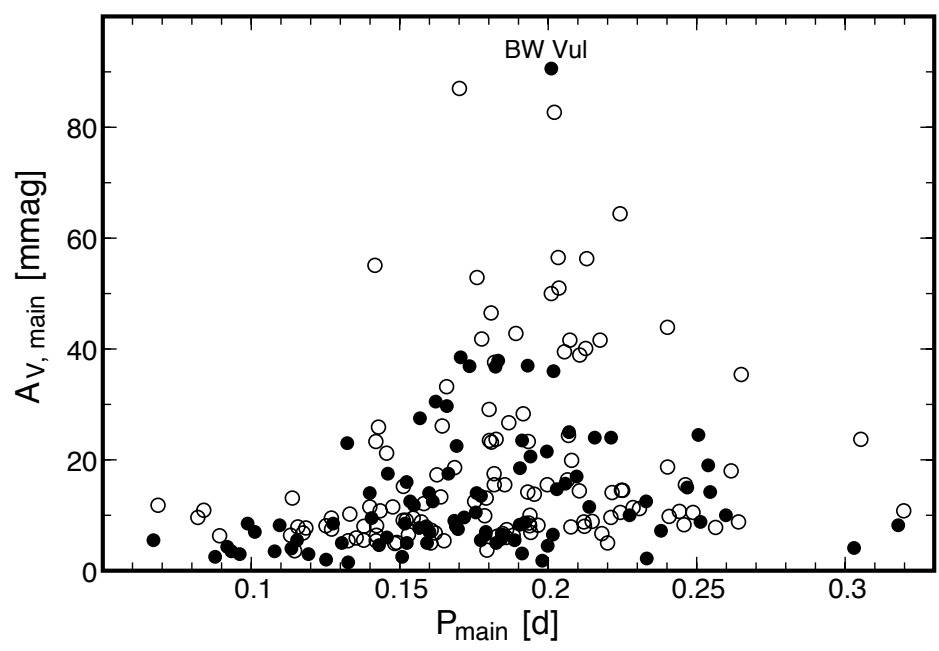

Figure 2: V-filter semi-amplitudes of the main mode, $A_{V \text {,main }}$, plotted as a function of the period of this mode, $\mathrm{P}_{\text {main }}$, for $220 \beta$ Cephei stars. Stars discovered in the ASAS data are shown with open circles, the other ones, as filled circles.

ASAS is not the only survey in which $\beta$ Cephei stars can be found. Narwid et al. (2006) found over 200 short-period variable stars in the OGLE-II photometry of Galactic fields. Without any doubts, many of them are $\beta$ Cephei stars. With the available photometry, however, they cannot be presently distinguished from $\delta$ Scuti stars. The new UBV photometry we are going to carry out for these stars will surely solve the problem. This will enable us to compare the pulsational properties of the $\beta$ Cephei stars located at larger distances and closer to the Galactic centre than the ASAS sample. The former stars are suspected to have higher metallicities than the latter which makes the comparison very attractive.

\section{Metallicity and pulsations of $\beta$ Cephei stars}

The previous sentence brings us to the problem of the dependence of the pulsations of $\beta$ Cephei stars on metallicity, predicted by theory. This dependence was already confirmed from the observations of Galactic open clusters (Pigulski et al. 2002), but later supported by the searches for $\beta$ Cephei stars in the Magellanic Clouds using the OGLE-II data (Kołaczkowski et al. 2004, 2006). The Magellanic Clouds have much smaller overall metallicities than our Galaxy and indeed the incidence of $\beta$ Cephei stars drops rapidly when going from our Galaxy to the Large (LMC) and then Small Magellanic Cloud. The study of these stars goes now to the spectroscopic work which should allow to establish observationally the lower limit of metallicity for the pulsations of $\beta$ Cephei stars.

\section{Conclusions}

Summarizing, there is indeed a rapid growth of the number of excellent data for $\beta$ Cephei stars that will allow statistical studies of these stars as a group. For example, there is a striking difference between the largest amplitudes of modes in $\beta$ Cephei stars located in the solar neighbourhood and those in the LMC (Kołaczkowski 2004). While for Galactic $\beta$ Cephei 
stars the semi-amplitudes reach 0.1 mag in $\mathrm{V}$ (Fig. 2), for all but two stars in the LMC they are smaller than $0.02 \mathrm{mag}$ in I which translates into less than 0.03 mag in $\mathrm{V}$. This may bring some clues for the long-standing problems of mode selection and amplitude limitation. Fortunately, some non-linear theoretical studies of $\beta$ Cephei stars have been recently undertaken (see Smolec \& Moskalik 2007). This and the growing number of asteroseismic studies can soon result not only in a better understanding of $\beta$ Cephei stars, but also in knowledge of the interiors of early B-type stars and pulsations in general.

Acknowledgments. This work was supported by the MNil grant 1 P03D 01627 . Many results presented here were obtained in cooperation with G. Pojmański (Warsaw Univ. Observatory), Z. Kołaczkowski and A. Narwid (Astronomical Institute, Univ. of Wroctaw). The author is grateful to the EC for the establishment of the European Helio- and Asteroseismology Network HELAS, which made his participation at this workshop possible.

\section{References}

Aerts C., Thoul A., Daszyńska J., et al., 2003, Sci, 300, 1926

Aerts C., De Cat P., Handler G., et al., 2004a, MNRAS, 347, 463

Aerts C., Waelkens C., Daszyńska-Daszkiewicz J., et al., 2004b, A\&A, 415, 241

Ausseloos M., Scuflaire R., Thoul A., Aerts C., 2004, MNRAS, 355, 352

Briquet M., Lefever K., Uytterhoeven K., Aerts C., 2005, MNRAS, 362, 619

Christensen-Dalsgaard J., 2002, Rev. Mod. Phys., 74, 1073

De Ridder J., Telting J. H., Balona L. A., et al., 2004, MNRAS, 351, 324

Dupret M.-A., Thoul A., Scuflaire R., et al., 2004, A\&A, 415, 251

Handler G., 2005, IBVS, 5667

Handler G., 2006, Comm. Asteroseis., 147, 31

Handler G., Shobbrook R. R., Jerzykiewicz M., et al., 2004, MNRAS, 347, 454

Handler G., Shobbrook R. R., Mokgwetsi, T., 2005, MNRAS, 362, 612

Handler G., Jerzykiewicz M., Rodríguez E., et al., 2006, MNRAS, 365, 327

Handler G., Tuvikene T., Lorenz D., et al., 2007, Comm. Asteroseis., 150, 193

Houk N., Swift C., 1999, Michigan Catalogue of Two-Dimensional Spectral Types for the HD Stars, Vol. 5, Univ. of Michigan Press, Ann Arbor, Michigan

Jerzykiewicz M., Handler G., Shobbrook R. R., et al., 2005, MNRAS, 360, 619

Kołaczkowski Z., 2004, Ph.D. thesis, University of Wrocław

Kołaczkowski Z., Pigulski A., Soszyński I., et al., 2004, in Kurtz D. W., Pollard K. R., eds, ASP Conf. Ser. Vol. 310, Variable Stars in the Local Group, IAU Colloquium 193. Astron. Soc. Pac., San Francisco, p. 225

Kołaczkowski Z., Pigulski A., Soszyński I., et al., 2006, Mem. Soc. Astron. Ital., 77, 336

Kurtz D. W., 2006, Comm. Asteroseis., 147, 6

Michel E., 2006, Comm. Asteroseis., 147, 40

Narwid A., Kołaczkowski Z., Pigulski A., 2006, Mem. Soc. Astron. Ital., 77, 342

Pamyatnykh A. A., Handler G., Dziembowski W. A., 2004, MNRAS, 350, 1022

Pigulski A., 2005, Acta Astron., 55, 219

Pigulski A., Kołaczkowski Z., Kopacki G., Jerzykiewicz M., 2002, in Aerts C., Bedding T. R., Christensen-Dalsgaard J., eds, ASP Conf. Ser. Vol. 259, Radial and Nonradial Pulsations as Probes of Stellar Physics. Astron. Soc. Pac., San Francisco, p. 146

Pigulski A., Handler G., Michalska G., et al., 2007, Comm. Asteroseis., 150, 191

Pojmański G., 2001, in Paczynski B., Chen W.-P., Lemme C., eds, ASP Conf. Ser. Vol. 246, Small Telescope Astronomy on Global Scales. Astron. Soc. Pac., San Francisco, p. 53

Pojmański G., Pilecki B., Szczygieł D., 2005, Acta Astron., 55, 275

Smolec R., Moskalik P., 2007, Comm. Asteroseis., 150, 205

Stankov A., Handler G., 2005, ApJS, 158, 193 


\section{DISCUSSION}

Mukadam: Do the high and low amplitude stars exhibit any other distinguishing properties?

Pigulski: High-amplitude stars are those with amplitudes larger than 40 mmag, but there is no difference for instance in luminosity. All $\beta$ Cephei stars have amplitudes lower than $0.1 \mathrm{mag}$.

Frandsen: There is also the super-WASP survey going on these days.

Pigulski: Yes, and there are other surveys, also in the Northern hemisphere and therefore I expect the amount of data and number of stars to increase rapidly over the next few years.

Aerts: Would you do a spectroscopic study of the eclipsing binary $\beta$ Cep stars, please?

Pigulski: Yes, this will be one of the first things we will do. We even have two of those stars in the same cluster, so we can do both of them at the same time.

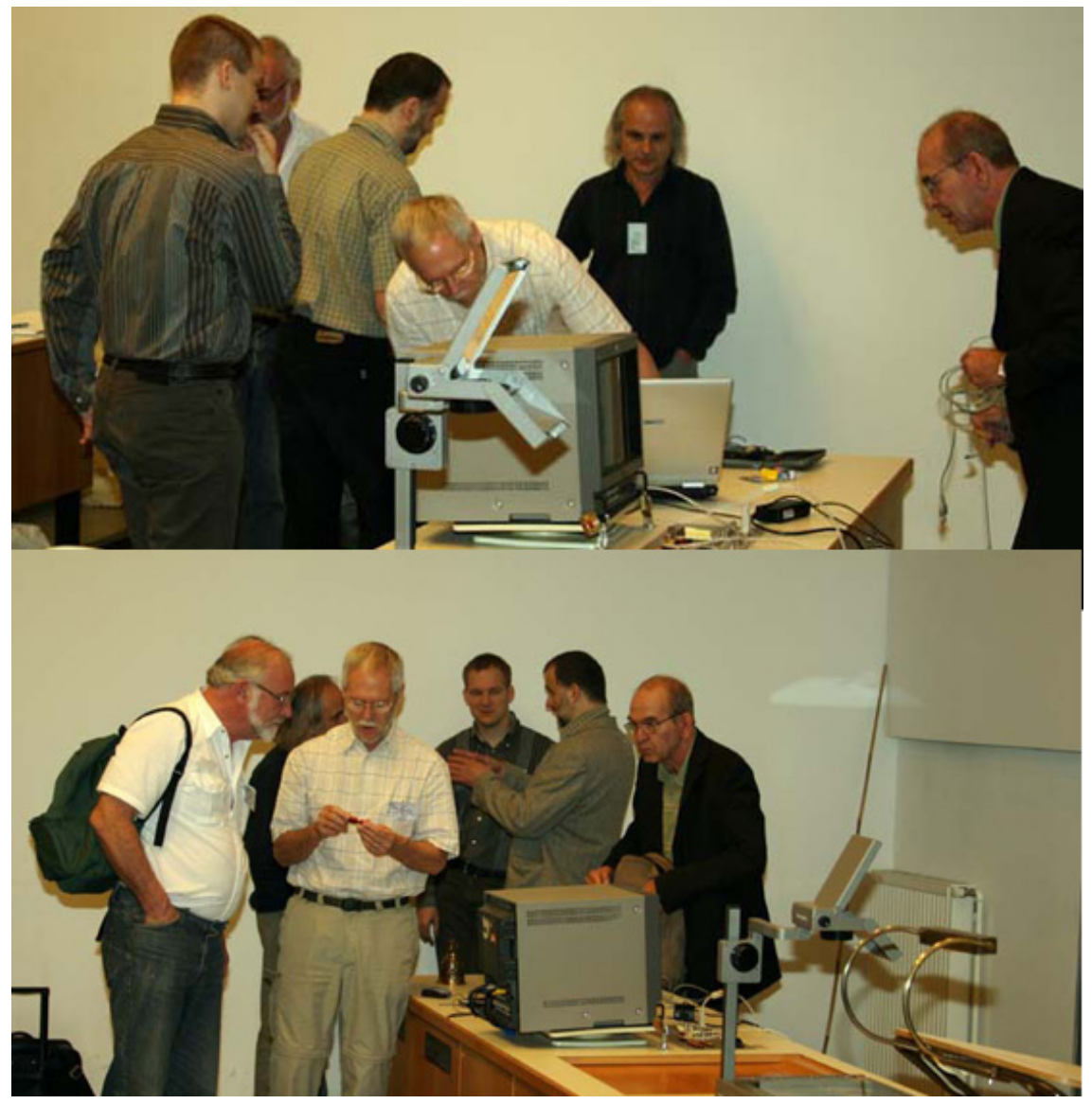

How many astronomers does it take to run a powerpoint presentation? 


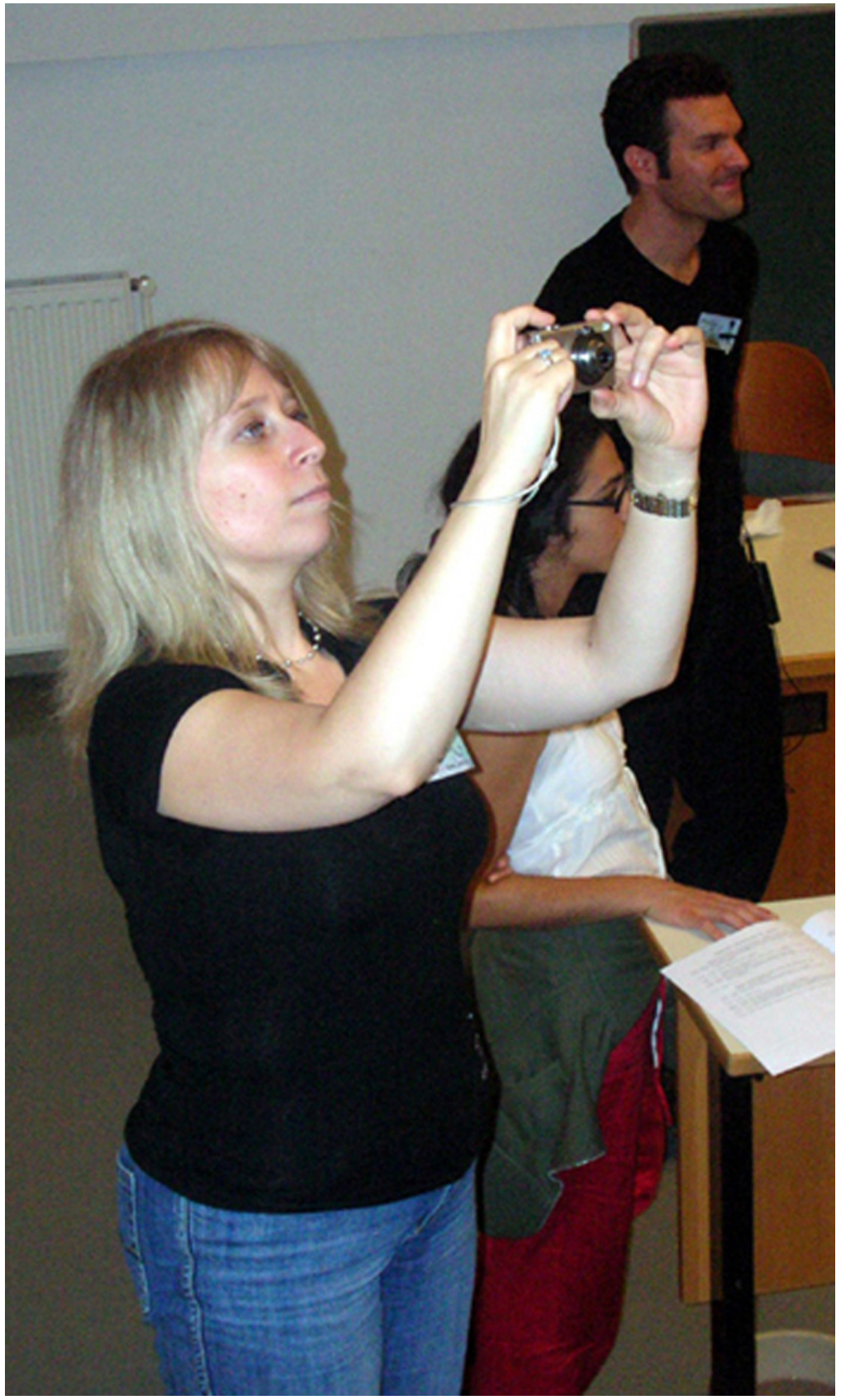

Konstanze Zwintz already working on these proceedings. 